\title{
Prompt production of the hidden charm pentaquarks in the LHC
}

\author{
Pan Ling ${ }^{1,2}$, Xiao-Hu Dai ${ }^{1,2}$, Meng-Lin Du ${ }^{3, a}$, Qian Wang ${ }^{1,2,4, b}$ \\ ${ }^{1}$ Guangdong Provincial Key Laboratory of Nuclear Science, Institute of Quantum Matter, South China Normal University, Guangzhou 510006, \\ China \\ ${ }^{2}$ Guangdong-Hong Kong Joint Laboratory of Quantum Matter, Southern Nuclear Science Computing Center, South China Normal University, \\ Guangzhou 510006, China \\ ${ }^{3}$ Instituto de Física Corpuscular (centro mixto CSIC-UV), Institutos de Investigación de Paterna, Apartado 22085, 46071 Valencia, Spain \\ ${ }^{4}$ Institute of High Energy Physics, Chinese Academy of Sciences, Beijing 100049, China
}

Received: 23 June 2021 / Accepted: 30 August 2021 / Published online: 16 September 2021

(C) The Author(s) 2021

\begin{abstract}
Motivated by the observation of the first hidden charm pentaquarks by the LHCb collaboration in 2015 and the updated analysis with an order-of-magnitude larger data set in 2019, we estimate their cross sections for the prompt production as well as their heavy quark spin partners, in the $\Sigma_{c}^{(*)} \bar{D}^{(*)}$ hadronic molecular picture, at the center-of-mass energy $7 \mathrm{TeV}$ in the $p p$ collision. Their cross sections are several $\mathrm{nb}$ and we would expect several tens hidden charm pentaquark events in the LHC based on its current integrated luminosity. The cross sections show a sizable deviation of the cross sections for hidden charm pentaquarks with the third isospin component $I_{z}=+\frac{1}{2}\left(P_{c}^{+}\right)$from those with $I_{z}=-\frac{1}{2}\left(P_{c}^{0}\right)$. The cross sections decrease dramatically with the increasing transverse momentum. Our study can also tell where to search for the missing hidden charm pentaquarks. The confirmation of the complete hidden charm pentaquarks in the heavy quark symmetry would further verify their $\Sigma_{c}^{(*)} \bar{D}^{(*)}$ molecular interpretation. In addition, the relative strength among these cross sections for pentaquarks can help us to identify the quantum numbers of the $P_{c}(4440)$ and $P_{c}(4457)$.
\end{abstract}

\section{Introduction}

The successful prediction of the $\Omega$ baryon has set a milestone of the conventional quark model, which hints the existence of the color degree of freedom and leads to the fundamental theory of the strong interaction, i.e. quantum chromodynamics (QCD). It is also a typical example of the connection between spectroscopy and underlying dynamics. The multiquark was first quantitatively studied by Jaffe in 1976 [1]

\footnotetext{
a e-mail: du.menglin@ific.uv.es

b e-mail: qianwang@m.scnu.edu.cn (corresponding author)
}

at the budding period of quark model. In the following tens of years, both theorists and experimentalists were committed to searching for the missing states and those beyond the conventional quark model, namely exotic states. The enthusiasm of studying multiquark states, however, was limited by the negative results and the low statistics of the experimental data. The situation broke until 2003 by the observation of the $D_{s 0}^{*}(2317)$ [2] and the $X$ (3872) [3]. As both the masses of the $D_{s 0}^{*}(2317)$ and the $X(3872)$ are significantly lower than its quark model expectation, they seriously challenged the conventional quark model and served as strong candidates for the exotic states. Up to now, tens of exotic candidates $[4,5]$ have been observed and various proposals were put forward about their configurations from theoretical side [6-16].

In a more general concept, all the boson (fermion) hadrons are classified to meson (baryon). The statistics of the experimental data for baryon sector is usually lower than that for the meson sector due to the number of quarks. Accordingly, the experimental data for exotic baryons are more scarce. Research enthusiasm is rekindled by the observation of the first hidden charm pentaquarks $P_{c}(4380)$ and $P_{c}(4450)$ in the $J / \psi p$ invariant mass distribution of the $\Lambda_{b} \rightarrow J / \psi p K^{-}$ process [17]. As they strongly decay into $J / \psi p$, they contain as least $c \bar{c} u u d$ five quarks unambiguously. Even before their observation, the hidden charm pentaquarks were proposed analogous to the excited baryons in light sector, e.g. in Refs. [18-24]. An updated analysis [25] of the LHCb Collaboration with an order-of-magnitude larger data set combined Run-I and II shows that the $P_{c}(4450)$ splits into two narrow peaks $P_{c}(4440)$ and $P_{c}(4457)$, and a third pentaquark $P_{c}(4312)$ emerges. Various interpretations follow this analysis, such as hadronic molecules [26-56], compact pentaquarks [51,5763], hadro-charmonia [64-66], and cusp effects [51]. Among these interpretations, the $\Sigma_{c}^{(*)} \bar{D}^{(*)}$ hadronic molecular pic- 
ture attracts the most attention based on the fact that the $P_{c}$ (4312) and $P_{c}(4440) / P_{c}(4457)$ are close to the $\Sigma_{c} \bar{D}$ and the $\Sigma_{c} \bar{D}^{*}$ thresholds, respectively. According to heavy quark spin symmetry (HQSS), there should exist seven hidden charm pentaquarks $[21,29,43,48,54,67-69]$, i.e. three $J^{P}=\frac{1}{2}^{-}$pentaquarks, three $J^{P}=\frac{3}{2}^{-}$pentaquarks and one $J^{P}=\frac{5}{2}^{-}$pentaquark with total angular momentum $J$ and parity $P$. Three of them are identified as the observed $P_{c}(4312)\left[\Sigma_{c} \bar{D}\right], P_{c}(4440)\left[\Sigma_{c} \bar{D}^{*}\right]$ and $P_{c}(4457)\left[\Sigma_{c} \bar{D}^{*}\right]$ $[29,43,48,54,67-69]$ by the LHCb Collaboration in 2019. By fitting to the $J / \psi p$ invariant mass spectrum, it is demonstrated in Refs. $[48,67]$ that the $\Sigma_{c}^{*} \bar{D}$ molecule should correspond to a new narrow $P_{c}(4380)$ which leaves a hint for its existence on the $J / \psi p$ distributions. However, the reason for almost invisibility of the $\Sigma_{c}^{*} \bar{D}^{*}$ molecules remains to be understood. It could be caused by the small production for the $\Sigma_{c}^{*} \bar{D}^{*}$ channel in the $\Lambda_{b}^{0}$ decay compared to other channels. Therefore, searching for the missing pentaquarks in different processes is a demanding task to complete the full spectrum and shed light on the underlying dynamics. On the other side, their closeness to the $\Sigma_{c}^{(*)} \bar{D}^{(*)}$ also indicates a large isospin violation in their decay rate, for instance the $P_{c}(4457) \rightarrow$ $J / \psi \Delta^{+}$process [28], and a significant deviation of the cross sections of the $P_{c}^{+}$pentaquarks with the third isospin component $I_{z}=+\frac{1}{2}$ from those of their isospin partners $P_{c}^{0}$ with $I_{z}=-\frac{1}{2}$.

Besides the $\Sigma_{c}^{(*)} \bar{D}^{(*)}$ hadronic molecular picture, the peaking structures in $J / \psi p$ distributions may be caused by kinematical effects, e.g. triangle singularities or cusps $[25,51,70]$. For instance, the $P_{c}(4457)$ might be generated by the $\Lambda_{c}^{+}(2590) \bar{D}^{0} D_{s}^{* *}$ triangle diagram [25]. The triangle singularities arise when all three hadrons in the trianglediagrams are nearly on mass shells and are manifested as peaks in the mass distributions [15]. Their manifestation are particularly sensitive to the momenta of incoming and outgoing particles. One of the conditions for the triangle singularities is that the mass of the decaying particle, i.e. $\Lambda_{b}^{0}$, should be very close to the threshold of the connected two internal particles. Once the deviation is larger, the triangle singularity condition will not be satisfied and the corresponding peaks will disappear. Thus searching for the hidden pentaquark states in the prompt production with a large incoming energy region will help to exclude the potential triangle singularity interpretation.

Motivated by the above arguments, we use Madgraph5 [71] and Phythia8 [72] to stimulate the prompt production rate of hidden charm pentaquarks in the hadronic molecular picture. Our framework is presented in Sect. 2. Results and discussions follows. A brief summary and outlook are given in the last section.

\section{Framework}

The inclusive production of a loosely bound $S$-wave hadronic molecule in hadron collision can be separated into a longrange part and a short-range part [73-77], ${ }^{1}$ which is based on the universal scattering amplitude for the low energy scattering. This kind of separation allows for estimating the cross section of the inclusive production for a given hadronic molecule, for instance the production of the $X(3872)[73,74]$ and its bottom analogs [77], the $D_{s 0}$ (2317) [75], the charged $Z_{c}^{(\prime) \pm}$ and $Z_{b}^{(\prime) \pm}$ [76]. We employ the formula presented in Refs. [76,77] to estimate the cross sections of inclusive prompt productions of hidden charm pentaquarks observed by LHCb in 2019.

\subsection{Factorization}

The production amplitude for the inclusive production of hidden charm pentaquarks, in the hadronic molecular picture, with small binding energy can be factorized as [76,77]

$$
\begin{aligned}
\mathcal{M}\left[P_{c}(E)\right] \approx & \sum_{\alpha} \int \frac{\mathrm{d}^{3} \mathbf{q}}{(2 \pi)^{3}} \mathcal{M}\left[\left(\Sigma_{c}^{(*)} \bar{D}^{(*)}\right)^{\alpha}(\mathbf{q})+\text { all }\right] \\
& \times G_{\alpha}(E, \mathbf{q}) \times T_{P_{c}}^{\alpha}(E),
\end{aligned}
$$

as illustrated by Fig. 1, with $\alpha$ the channel index. This factorization is based on the separation of the involved scales, i.e. the binding momentum $\sqrt{2 \mu E_{B}}$ with $\mu$ the reduced mass of $\Sigma_{c}^{(*)} \bar{D}^{(*)}$ and $E_{B}$ the binding energy from the large scale of QCD for point-like production in the effective field theory point of view. While the inclusive production of the $\Sigma_{c}^{(*)} \bar{D}^{(*)}$ from $p p$ collision and the formation of the loosely bound molecular hidden charm pentaquarks are regarded as a shortrange interaction and a long-range interaction, respectively. This idea is proposed in Refs. [73,74] and has been used to estimate the production of heavy quarkonium-like states in Refs. [76,77,79-82] and charm-strange molecules in Ref. [75]. ${ }^{2}$ In this work, since we only aim at an estimate of orderof-magnitude, only the production through $S$-wave $\Sigma_{c}^{(*)} \bar{D}^{(*)}$ channels are considered. Here $\mathcal{M}\left[\left(\Sigma_{c}^{(*)} \bar{D}^{(*)}\right)^{\alpha}+\right.$ all $]$ is the production amplitude of the corresponding constituent for a given pentaquark. $T^{\alpha}$ is the amplitude for the $\Sigma_{c}^{(*)} \bar{D}^{(*)}$ to the $P_{c}$ pentaquark.

$G_{\alpha}(E, \mathbf{q})=\frac{2 \mu_{\alpha}}{q^{2}-p_{\alpha}^{2}-i \epsilon}$,

is the intermediated two-body propagator, with $p_{\alpha}$ the nonrelativistic three momentum of the $\alpha$ th channel, extracting

\footnotetext{
1 The calculation in this work is based on the $\Sigma_{c}^{(*)} \bar{D}^{(*)}$ molecular picture. The analogous calculation can also be done for the compact pentaquarks similar to the production of the $X(3872)$ in $p \bar{p}$ collision [78].

2 The debate of the yield of the $X(3872)$ at high $p_{T}$ can be found in Refs. [78,80,83].
} 


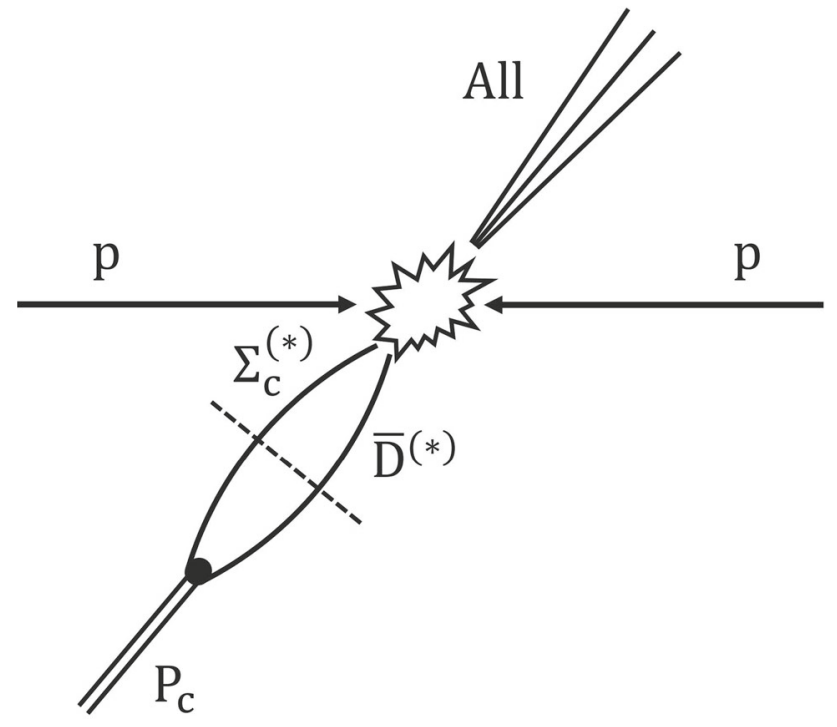

Fig. 1 The factorization of the inclusive production of hidden charm pentaquarks in $p p$ collision in the $\Sigma_{c}^{(*)} \bar{D}^{(*)}$ hadronic molecular picture. The other particles produced with the $\Sigma_{c}^{(*)} \bar{D}^{(*)}$ are denoted as "All" in the figure

from below equation

$p_{\alpha}^{2} \equiv 2 \mu_{\alpha}\left(E-m_{\mathrm{th}}^{\alpha}\right)$.

Here $\mu_{\alpha}, m_{\text {th }}^{\alpha}$ and $E$ are the reduced mass, threshold of the $\alpha$ th channel and the total energy, respectively. In low energy, we are interested in, to the leading order, the amplitudes $\mathcal{M}\left[\left(\Sigma_{c}^{(*)} \bar{D}^{(*)}\right)^{\alpha}+\right.$ all $]$ and $T^{\alpha}$ could be treated as constants [76,77], leaving the equation reduced into an algebraic equation. As the result, we only need to calculate the integration

$$
\begin{aligned}
\int \frac{\mathrm{d}^{3} \boldsymbol{q}}{(2 \pi)^{3}} G_{\alpha}(E, \mathbf{q}) & =\int \frac{\mathrm{d}^{3} \boldsymbol{q}}{(2 \pi)^{3}} \frac{2 \mu_{\alpha}}{\mathbf{q}^{2}-p_{\alpha}^{2}-i \epsilon} \\
& =\int \frac{q^{2} \mathrm{~d} q}{2 \pi^{2}} \frac{2 \mu_{\alpha}}{q^{2}-p_{\alpha}^{2}-i \epsilon},
\end{aligned}
$$

which is linearly divergent and needs to be regularized. To that end, a hard cut-off $\Lambda$ is introduced to render the integral well defined

$$
\begin{aligned}
\mathcal{G}_{\alpha}(E) & :=\int_{0}^{\Lambda} \frac{q^{2} \mathrm{~d} q}{2 \pi^{2}} \frac{2 \mu_{\alpha}}{q^{2}-p_{\alpha}^{2}-i \epsilon} \\
& =\frac{\mu_{\alpha} \Lambda}{\pi^{2}}-\frac{\mu_{\alpha} p_{\alpha}}{\pi^{2}} \operatorname{ArcTanh}\left(\frac{p_{\alpha}}{\Lambda}\right)+i \frac{\mu_{\alpha} p_{\alpha}}{\pi} .
\end{aligned}
$$

The value of $\Lambda$ is determined by the effectiveness of the low energy theorem which inherits the non-perturbative mechanism of strong interaction and is of order of $1 \mathrm{GeV}$. In our case, we take values $\Lambda=[0.7,1.3] \mathrm{GeV}$ to estimate the cross sections. $^{3}$

\footnotetext{
3 The lower limit $0.7 \mathrm{GeV}$ should be larger than the largest three momentum of the involved dynamic channels. The upper limit $1.3 \mathrm{GeV}$
}

Before going into the production of the $P_{c}$ states, the cross section of the inclusive $\Sigma_{c}^{(*)} \bar{D}^{(*)}$ production should be estimated by Monte Carlo (MC) simulation and reads as

$$
\begin{aligned}
& \mathrm{d} \sigma\left[\Sigma_{c}^{(*)} \bar{D}^{(*)}\right]_{M C}=K_{\Sigma_{c}^{(*)}} \bar{D}^{(*)} \frac{1}{\text { flux }} \sum_{\text {all }} \mathrm{d} \phi_{\Sigma_{c}^{(*)} \bar{D}^{(*)}+\text { all }} \\
& \quad \times \mid\left.\mathcal{M}\left[\Sigma_{c}^{(*)} \bar{D}^{(*)}+\text { all }\right]\right|^{2} \frac{\mathrm{d}^{3} k}{(2 \pi)^{3} 2 \mu},
\end{aligned}
$$

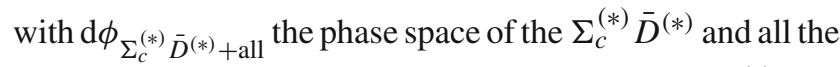
other particles. $k$ is the relative momentum between $\Sigma_{c}^{(*)} \bar{D}^{(*)}$ in its center-of-mass frame. $K_{\Sigma_{c}^{(*)} \bar{D}^{(*)}}$ is a normalization factor to compensate the difference between the MC and experimental data, and is taken the value 1 as an order-of-magnitude estimate. In total, the cross section of the inclusive $P_{c}$ production can be written as

$$
\begin{aligned}
& \sigma\left[P_{c}\right]=\sum_{\alpha} \frac{1}{4 m_{\Sigma_{c}^{(*)}} m_{\bar{D}^{(*)}}}\left|\tilde{g}_{\alpha}^{P_{c}}\right|^{2}\left|\mathcal{G}_{\alpha}\right|^{2} \\
& \times\left(\frac{\mathrm{d} \sigma\left[\left(\Sigma_{c}^{(*)} \bar{D}^{(*)}(k)\right)^{\alpha}\right]}{\mathrm{d} k}\right)_{M C} \frac{4 \pi^{2} \mu_{\alpha}}{k^{2}},
\end{aligned}
$$

where

$\left|\tilde{g}_{\alpha}^{P_{c}}\right|^{2}=\frac{\left(2 J_{P_{c}}+1\right)\left|g_{\alpha}^{P_{c}}\right|^{2}}{\sum_{i}\left(2 J_{i}+1\right)\left|g_{\alpha}^{i}\right|^{2}}$

means the fraction of the $\alpha$ th channel events to a given $P_{c}$ with spin $J_{P_{c}}$. The sum $i$ in the denominator runs all the pentaquark states which couple to the $\alpha$ th channel. $g_{\alpha}$ is the coupling of the $\alpha$ th channel to a given $P_{c}$ state and the values can be found in Appendix A.

\subsection{Monte Carlo simulation}

The production of hidden charm pentaquarks in the hadronic molecular picture should follow the production of the corresponding constituents, i.e. the heavy quark pair $c \bar{c}$ in the parton level. Considering the other produced particles in the inclusive process, a third parton should be produced simultaneously. As the result, the $2 \rightarrow 3$ parton process should be generated through hard scattering and hadronized into final hadrons via non-perturbative mechanism.

Similar to those in Refs. [76,77], we generate the $2 \rightarrow 3$ process via Madgraph5 [71] and use Phythia8 [72] for the hadronization. As the two constituents $\Sigma_{c}^{(*)} \bar{D}^{(*)}$ should be collinear and with relative small momentum, the cut $p_{\mathrm{T}}>$ $3.5 \mathrm{GeV}$ and $|y|<2$ are implemented for the heavy quark pair. In principle, all the parton level $2 \rightarrow 3$ processes will contribute. However, we demonstrate numerically, as shown

is largest value of $\Lambda$ making the physical observables renormalization group invariant [67]. 


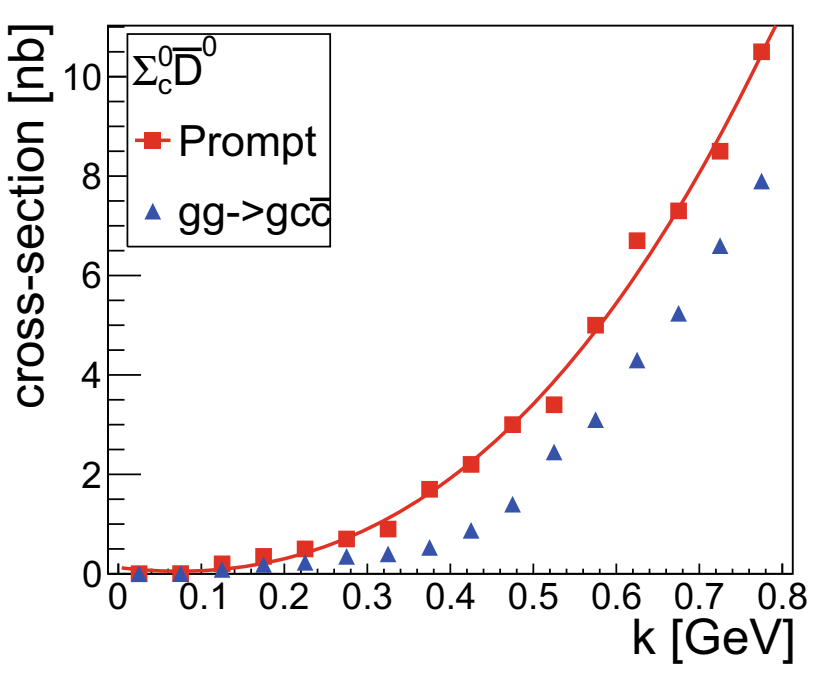

Fig. 2 The cross section of the inclusive $\Sigma_{c}^{0} \bar{D}^{0}$ pair production in terms of their relative momentum at the center-of-mass energy $\sqrt{s}=7 \mathrm{TeV}$. The red box points are obtained by all the parton level diagrams switched on. The blue triangle points are those for only the $g g \rightarrow g c \bar{c}$ switched on. The red sold curve is an interpolation. The case for other $\Sigma_{c}^{(*)} \bar{D}^{(*)}$ channel is the same, i.e. the $g g \rightarrow g c \bar{c}$ parton process is the most important contribution

in Fig. 2, that the $g g \rightarrow g c \bar{c}^{4}$ is the most important parton process similar to its contribution in the $X(3872)$ production $[73,74]$. Accordingly, to improve the efficiency of the code, only the $g g \rightarrow g c \bar{c}$ process is considered in Madgraph5. In addition, the dependence on the relative momentum $k$ is $[76,77]$

$\frac{\mathrm{d} \sigma\left[\Sigma_{c}^{(*)} \bar{D}^{(*)}(k)\right]_{\mathrm{MC}}}{\mathrm{d} k} \sim k^{2}$

at low energy without considering the final-state-interaction (FSI).

As the hadronization process is still unclear and modeldependent, the hadronization implemented in Phythia8 [72] is incomplete and we might underestimate the yield of heavy hadron pairs. A comparison between the MC simulation results, with all the parton processes considered, of the $D^{0}$ meson and the $\Lambda_{c}^{+}$charm baryon ${ }^{5}$ with the experimental data are presented in Fig. 3. Although the experimental data and the MC simulation result are of the same order, the deviation is still sizable, especially for the charmed baryon. The deviation is because of the missing dynamics in Phythia8, for instance the feed-down charm meson/baryon from bottom meson/baryon [86]. As we only make an order-of-magnitude estimate for the cross sections, this deviation can be accepted.

\footnotetext{
4 To estimate the uncertainty of this approximation, we compare the $\Sigma_{c}^{+} \bar{D}^{0}$ inclusive cross sections within these two frameworks and find that the deviation is under $5 \%$.

5 As the cross section of the $\Sigma_{c}$ charmed baryon is not existing, we use that of the $\Lambda_{c}^{+}$as an illustration for the production of charm baryons.
}

\section{Results and discussions}

As discussed in the introduction, the closeness of the $P_{c} \mathrm{~s}$ to the $\Sigma_{c}^{(*)} \bar{D}^{(*)}$ threshold might indicate a significant deviation of the cross sections of the $P_{c}^{+}$from those of the $P_{c}^{0}$, we explicitly consider the cross sections of the inclusive productions of $P_{c}^{+}$and $P_{c}^{0}$ with wave functions ${ }^{6}$

$$
\begin{aligned}
P_{c}^{+} & =-\sqrt{\frac{1}{3}} \Sigma_{c}^{(*)+} \bar{D}^{(*) 0}+\sqrt{\frac{2}{3} \Sigma_{c}^{(*)++} D^{(*)-},} \\
P_{c}^{0} & =\sqrt{\frac{1}{3}} \Sigma_{c}^{(*)+} D^{(*)-}-\sqrt{\frac{2}{3}} \Sigma_{c}^{(*) 0} \bar{D}^{(*) 0},
\end{aligned}
$$

in the hadronic molecular picture. To study the deviation quantitatively, the ratio

$\operatorname{Ratio}\left(P_{c}^{+} / P_{c}^{0}\right) \equiv \frac{\mathrm{d} \sigma\left(P_{c}^{+}\right)}{\mathrm{d} p_{\mathrm{T}}} / \frac{\mathrm{d} \sigma\left(P_{c}^{0}\right)}{\mathrm{d} p_{\mathrm{T}}}$

between the cross sections of the $P_{c}^{+}$and $P_{c}^{0}$ is also defined, where the statistic uncertainties have been cancelled out. The cross sections and the corresponding ratios for the inclusive production of the $P_{c}$ s in $p p$ collision at center-of-mass energy $\sqrt{s}=7 \mathrm{TeV}$ are presented in Fig. 4. The left and right panels in Fig. 4 are for the two solutions, denoted as A and B, in the molecular picture $[29,43,48,67]$. In Solution A, the $P_{c}(4440)$ and the $P_{c}(4457)$ are assigned as $\frac{1}{2}^{-}$and $\frac{3}{2}^{-}$pentaquarks, respectively. In Solution $\mathrm{B}$, they are interchanged. From Fig. 4, one can see that the production rates decrease dramatically with the increasing $p_{\mathrm{T}}$, which stems from the decreasing behavior of the fragmentation functions [73]. The significant deviations of the cross sections of the charged $P_{c}^{+} \mathrm{s}$ from those of the neutral ones $P_{c}^{0}$ s can also be seen directly from cross section of their constituents in Appendix B. The total cross sections are several nb as shown in Table 1 and Fig. 5, with the lower and upper limits corresponding to those with $\Lambda=0.7 \mathrm{GeV}$ and $\Lambda=1.3 \mathrm{GeV}$ as an order-ofmagnitude estimate. Considering the integrated luminosity $\mathcal{L}=34 \mathrm{nb}^{-1}$ [87] of LHC at $\sqrt{s}=7 \mathrm{TeV}$, we would expect to collect several tens $P_{c}$ s events in LHC. Furthermore, the observation of the narrow $P_{c}(4380)[48,67]$ and the $P_{c}$ s relevant to the $\Sigma_{c}^{*} \bar{D}^{*}$ channel will complete the spectroscopy of the hidden charm pentaquarks of the $\Sigma_{c}^{(*)} \bar{D}^{(*)}$ hadronic molecular picture [29,43,48,54,67-69].

The result of two solutions for the $P_{c}$ productions are collected in Table 1 and Fig. 5. In both solutions, the cross section for $P_{c}$ (4380) is the largest one due to the largest production of $\Sigma_{c}^{*} \bar{D}$ channel (Eq. (B1)) and its strong coupling to $P_{c}(4380)$, which makes the prompt production in $p p$ colli-

\footnotetext{
${ }^{6}$ The third isospin components of $P_{c}^{+}$and $P_{c}^{0}$ are $I_{z}=+\frac{1}{2}$ and $I_{z}=$ $-\frac{1}{2}$, respectively. In what follows, if the charged property of the hidden charm pentaquarks is not specified, the argument works for both of them.
} 


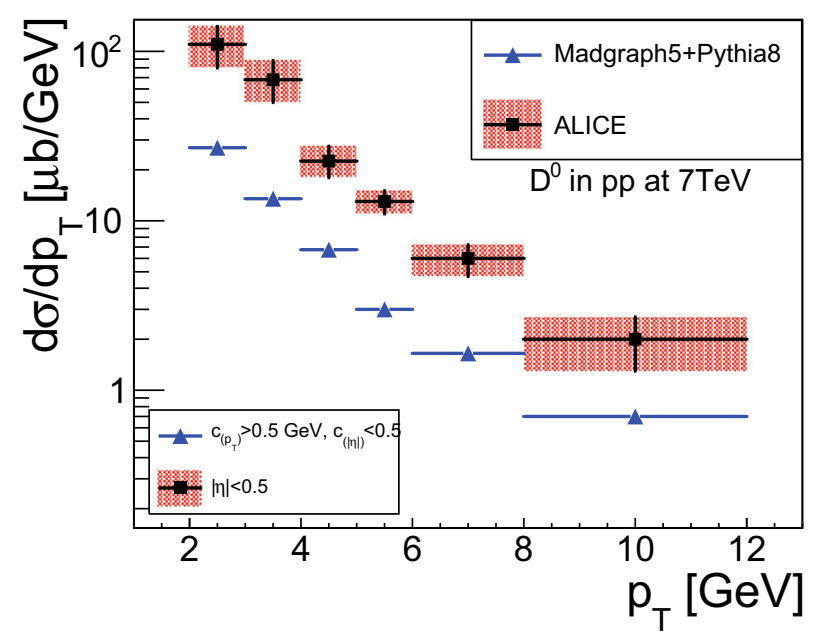

Fig. $3 \mathrm{MC}$ simulation of the cross section of the inclusive $D^{0}$ (left) and $\Lambda_{c}^{+}$(right) production in the $p p$ collision at $\sqrt{s}=7 \mathrm{TeV}$ comparing with the experimental data from Refs. $[84,85]$. The rapidity

sion an ideal platform for the search of the narrow $P_{c}(4380)$ $[48,67]$. For $J^{P}=1 / 2^{-}$channel,

$\sigma_{\mathrm{A}}\left(P_{c}(4312)\right)>\sigma_{\mathrm{A}}\left(P_{c}(4440)\right)>\sigma_{\mathrm{A}}\left(P_{c}\left(\frac{1}{2}^{-}\right)\right)$,

$\sigma_{\mathrm{B}}\left(P_{c}(4312)\right)>\sigma_{\mathrm{B}}\left(P_{c}(4457)\right)>\sigma_{\mathrm{B}}\left(P_{c}\left(\frac{1}{2}^{-}\right)\right)$,

with subindexes A and B for the two solutions. As they mainly couple to the $\Sigma_{c} \bar{D}, \Sigma_{c} \bar{D}^{*}, \Sigma_{c}^{*} \bar{D}^{*}$ channels, respectively, the relations Eqs. (13) and (14) are largely determined by the cross sections of their constituents

$\sigma\left(\Sigma_{c} \bar{D}\right)>\sigma\left(\Sigma_{c} \bar{D}^{*}\right) \simeq \sigma\left(\Sigma_{c}^{*} \bar{D}^{*}\right)$.

For the $\Sigma_{c}^{*} \bar{D}^{*}$ relevant $P_{c}$ states, a further suppressed factor comes from the denominator of Eq. (8), i.e. three $\frac{1}{2}^{-}$, three $\frac{3}{2}^{-}$and one $\frac{5}{2}^{-}$states coupling to the same channel. In addition, one can also see the relation

$\sigma\left(P_{c}(4440)\right)>\sigma\left(P_{c}(4457)\right)$

in both solutions. That is because the cross section of a state is proportional to the absolute square of its coupling to the constitutes and thus its binding energy for shallow bound states [11].

From Table 1 and Fig. 5, one can also see different patterns of the prompt cross sections for Solutions A and B which can be used to distinguish the two solutions. It is noticed that the productions of the $P_{c}$ states are sensitive to the cross sections of the $\Sigma_{c}^{(*)} \bar{D}^{(*)}$ which however receive large uncertainties due to the unknown hadronisation mechanism. To some extend, the results in Table 1 and Fig. 5 are understood

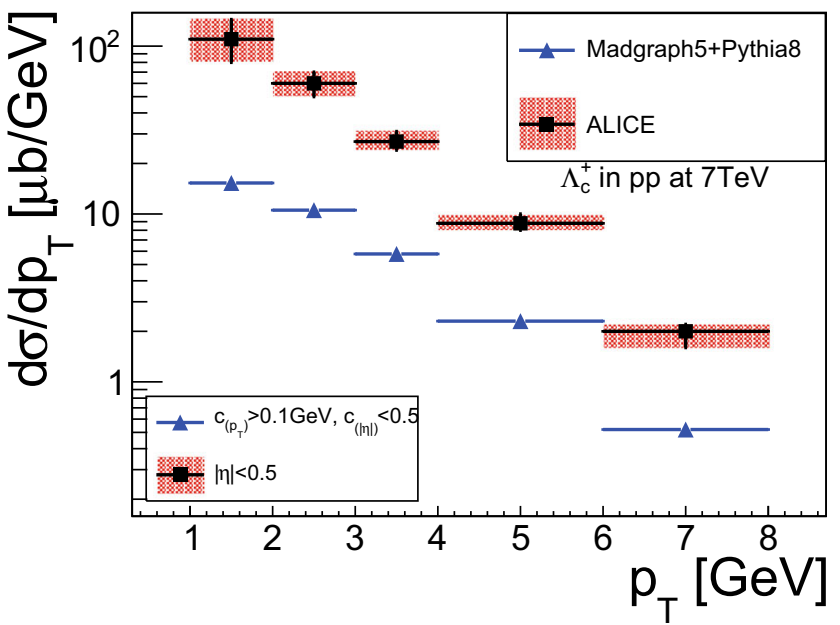

cut $|\eta|<0.5$ is implemented for charm quark to compare with the experimental data. The black box and blue triangle points are for the experimental data and $\mathrm{MC}$ results, respectively

as an order-of-magnitude estimate and a comparison of the cross sections between different $P_{c}$ states should be made with great care. Among them the situation for the $J^{P}=\frac{5}{2}^{-}$ pentaquark is the most simple one, as it only couples to the $\Sigma_{c}^{*} \bar{D}^{*}$ channel. That the cross section in Solution A is much smaller than that in Solution B is due to the smaller distance of their pole positions to the $\Sigma_{c}^{*} \bar{D}^{*}$ threshold and the resulting smaller effective coupling. An important observation is that the three $\Sigma_{c}^{*} \bar{D}^{*}$ molecular states exhibit different patterns in two solutions. For Solution B, the state with higher spin has a larger binding energy, and thus effective coupling. Combined with the enhancing factor $(2 J+1)$ in Eq. (8) for higher spins, it leads to a significant relation, see e.g. in Fig. 5,

$\sigma_{\mathrm{B}}\left(P_{c}\left(\frac{1}{2}^{-}\right)\right)<\sigma_{\mathrm{B}}\left(P_{c}\left(\frac{3}{2}^{-}\right)\right)<\sigma_{\mathrm{B}}\left(P_{c}\left(\frac{5}{2}^{-}\right)\right)$.

However, the enhancing factor for the higher spin is roughly balanced by the smaller effective coupling in Solution A, which makes the cross sections for the three states comparable. We stress that this difference is independent on the production of $\Sigma_{c}^{(*)} \bar{D}^{(*)}$ and only relies on the mass of pattern of the three $\Sigma_{c}^{*} \bar{D}^{*}$ states. It provides us an important way to distinguish the two solutions, as well as to identify the quantum numbers of the $P_{c}(4440)$ and $P_{c}(4457)$ since they are assigned to $J^{P}=\frac{1}{2}^{-}$and $\frac{3}{2}^{-}$in Solution $\mathrm{A}$ and interchanged in Solution B. 
Fig. 4 The cross sections of the inclusive charged hidden charm pentaquarks, i.e. the charged $P_{c}^{+} \mathrm{s}$, in $p p$ collision at $\sqrt{s}=7 \mathrm{TeV}$ in terms of the transverse momentum $p_{\mathrm{T}}$ with $\Lambda=0.7 \mathrm{GeV}$. Their ratios relative to the neutral ones, as defined in Eq. (12), are also presented below each figure. The three figures on the left (a, $\mathbf{c}, \mathbf{e})$ and right $(\mathbf{b}, \mathbf{d}, \mathbf{f})$ hand sides are for the solution $\mathrm{A}$ and $B$ in Ref. [67], respectively. Figures $\mathbf{a}, \mathbf{b}$ are for the $P_{c} \mathrm{~s}$ with quantum number $J^{P}=\frac{1}{2}^{-}$. Figures $\mathbf{c}, \mathbf{d}$ are for the $P_{c}$ s with quantum number $J^{P}=\frac{3}{2}^{-}$. Figures $\mathbf{e}, \mathbf{f}$ are for the $P_{c} \mathrm{~s}$ with quantum number $J^{P}=\frac{5}{2}^{-}$. In each figure, the $P_{c}$ s are labeled, from lower to higher mass, as red box, black inverted triangle and blue triangle
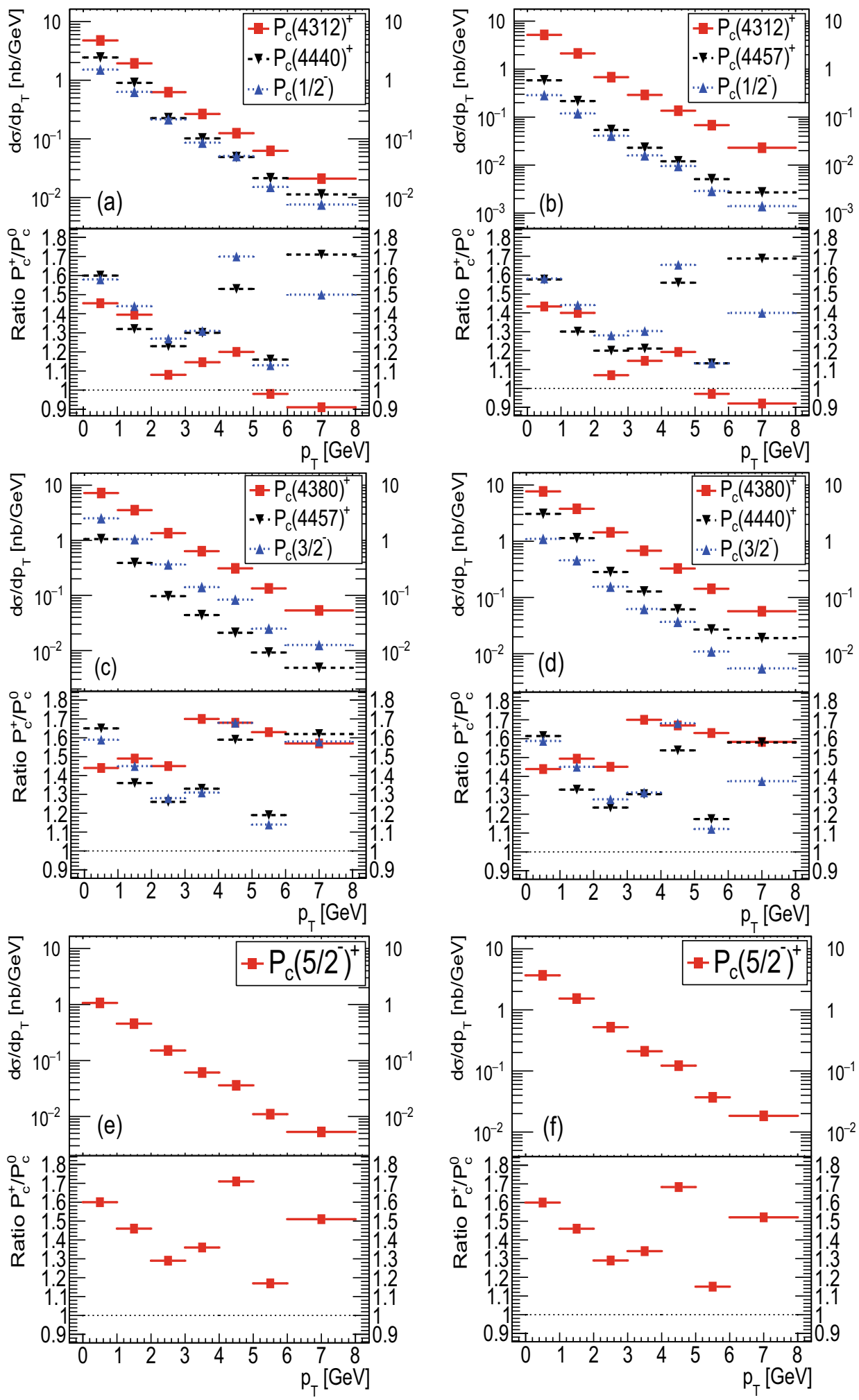
Table 1 The estimate of the inclusive cross section of hidden charm pentaquark states $P_{c}^{+} \mathrm{s}$ and $P_{c}^{0} \mathrm{~s}$ in $p p$ collision at $\sqrt{s}=7$ TeV with the lower and upper limits corresponding to $\Lambda=0.7 \mathrm{GeV}$ and $\Lambda=1.3 \mathrm{GeV}$, respectively

\begin{tabular}{|c|c|c|c|c|c|c|}
\hline \multirow[t]{2}{*}{$J^{P}$} & \multicolumn{3}{|l|}{ Solution A } & \multicolumn{3}{|l|}{ Solution B } \\
\hline & States & $P_{c}^{+}(\mathrm{nb})$ & $P_{c}^{0}(\mathrm{nb})$ & States & $P_{c}^{+}(\mathrm{nb})$ & $P_{c}^{0}(\mathrm{nb})$ \\
\hline \multirow[t]{3}{*}{$\frac{1}{2}^{-}$} & $P_{c}(4312)$ & $3.49 \sim 7.82$ & $2.53 \sim 5.79$ & $P_{c}(4312)$ & $3.94 \sim 8.50$ & $2.88 \sim 6.33$ \\
\hline & $P_{c}(4440)$ & $2.04 \sim 3.76$ & $1.42 \sim 2.63$ & $P_{c}(4457)$ & $0.45 \sim 0.90$ & $0.32 \sim 0.64$ \\
\hline & $P_{c}\left(\frac{1}{2}^{-}\right)$ & $1.44 \sim 2.54$ & $0.95 \sim 1.69$ & $P_{c}\left(\frac{1}{2}^{-}\right)$ & $0.24 \sim 0.48$ & $0.16 \sim 0.32$ \\
\hline \multirow[t]{3}{*}{$\frac{3}{2}^{-}$} & $P_{c}(4380)$ & $6.23 \sim 13.35$ & $4.20 \sim 9.09$ & $P_{c}(4380)$ & $6.85 \sim 14.26$ & $4.63 \sim 9.73$ \\
\hline & $P_{c}(4457)$ & $0.73 \sim 1.62$ & $0.48 \sim 1.09$ & $P_{c}(4440)$ & $2.50 \sim 4.73$ & $1.72 \sim 3.28$ \\
\hline & $P_{c}\left(\frac{3}{2}^{-}\right)$ & $2.34 \sim 4.19$ & $1.55 \sim 2.78$ & $P_{c}\left(\frac{3}{2}^{-}\right)$ & $0.98 \sim 1.84$ & $0.65 \sim 1.22$ \\
\hline$\frac{5}{2}^{-}$ & $P_{c}\left(\frac{5}{2}^{-}\right)$ & $0.81 \sim 1.78$ & $0.50 \sim 1.17$ & $P_{c}\left(\frac{5}{2}^{-}\right)$ & $3.30 \sim 6.12$ & $2.16 \sim 4.03$ \\
\hline
\end{tabular}

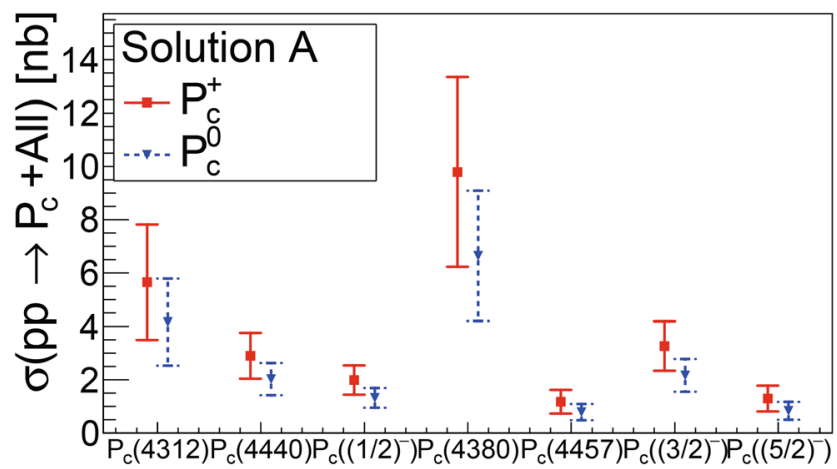

Fig. 5 The cross section of the prompt production of the hidden charm pentaquarks in the $p p$ collision at $\sqrt{s}=7 \mathrm{TeV}$. The red boxes and blue inverse triangles are for the charged pentaquarks $P_{c}^{+}$and the neutral ones $P_{c}^{0}$, respectively. The left and right panels are for the solution A and solution B in the hadronic molecular picture. The lower limits, the upper ones and the central values correspond to the cross sections with

\section{Summary}

In a short summary, within the $\Sigma_{c}^{(*)} \bar{D}^{(*)}$ hadronic molecular picture, we estimate the prompt cross sections of the seven hidden charm pentaquarks at center-of-mass energy $\sqrt{s}=7 \mathrm{TeV}$ in the $p p$ collision. The observation of the narrow $P_{c}$ (4380) and the $P_{c}$ s related to the $\Sigma_{c}^{*} \bar{D}^{*}$ threshold, which are insignificant in the $\Lambda_{b}$ decay, will complete the spectroscopy in the hadronic molecular picture. That will confirm their $\Sigma_{c}^{(*)} \bar{D}^{(*)}$ molecular picture. Their cross sections are several nb and one would expect several tens events based on the current integrated luminosity of LHC at $\sqrt{s}=7 \mathrm{TeV}$. The cross sections decrease dramatically with the increasing transverse momentum due to the decreasing behavior of fragmentation functions. In addition, as these seven hidden charm pentaquarks are close to the corresponding thresholds, there are also sizable deviations of the charged $P_{c}^{+}$cross sections from those of the neutral ones. The different patterns of the cross sections could be used to distinguish the two solutions in the molecular picture, which will help

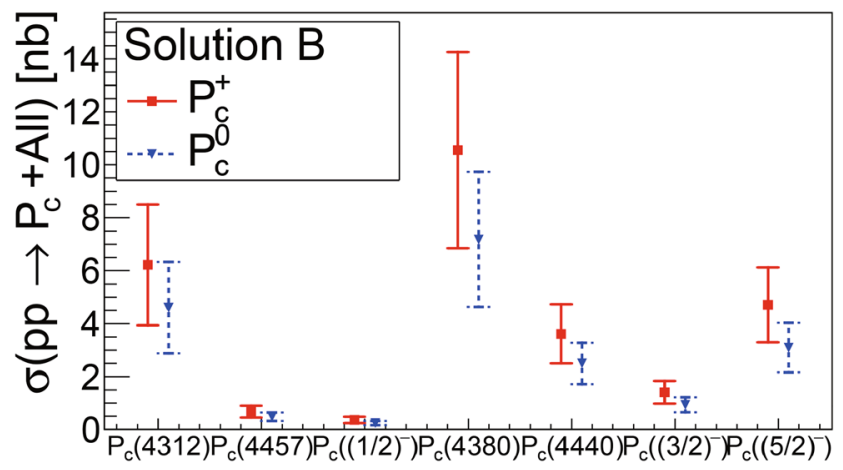

$\Lambda=0.7 \mathrm{GeV}, \Lambda=1.3 \mathrm{GeV}$ and their average values. The difference of uncertainties are mainly because of the $k$-dependence of the heavy hadron pairs cross section, e.g. Eq. (9) in MC simulation. In our calculation, the $k$-dependence in each $p_{\mathrm{T}}$ bin is evaluated numerically, but not the simple $k^{2}$ behavior

us to identify the quantum numbers of the $P_{c}(4440)$ and $P_{c}$ (4457).

Acknowledgements The discussions with Eulogio Oset, Feng-Kun Guo and Hongxi Xing are appreciated. This work is partly supported by Guangdong Major Project of Basic and Applied Basic Research no. 2020B0301030008, the National Natural Science Foundation of China with Grant no. 12035007, Science and Technology Program of Guangzhou No. 2019050001, Guangdong Provincial funding with Grant no. 2019QN01X172. Q.W. is also supported by the by the NSFC and the Deutsche Forschungsgemeinschaft (DFG, German Research Foundation) through the funds provided to the Sino-German Collaborative Research Center TRR110 "Symmetries and the Emergence of Structure in QCD" (NSFC Grant no. 12070131001, DFG Project-ID 196253076-TRR 110). The work of M.L.D. is supported by the Spanish Ministerio de Economía y Competitividad (MINECO) and the European Regional Development Fund (ERDF) under contract FIS2017-84038C2-1-P, by the EU Horizon 2020 research and innovation programme, STRONG-2020 project, under Grant agreement no. 824093, by Generalitat Valenciana under contract PROMETEO/2020/023.

Data Availability Statement This manuscript has no associated data or the data will not be deposited. [Authors' comment: All the relevant data and the related references have already been given in the paper.] 
Open Access This article is licensed under a Creative Commons Attribution 4.0 International License, which permits use, sharing, adaptation, distribution and reproduction in any medium or format, as long as you give appropriate credit to the original author(s) and the source, provide a link to the Creative Commons licence, and indicate if changes were made. The images or other third party material in this article are included in the article's Creative Commons licence, unless indicated otherwise in a credit line to the material. If material is not included in the article's Creative Commons licence and your intended use is not permitted by statutory regulation or exceeds the permitted use, you will need to obtain permission directly from the copyright holder. To view a copy of this licence, visit http://creativecomm ons.org/licenses/by/4.0/.

Funded by SCOAP ${ }^{3}$.

\section{Appendix A: The effective couplings of the hidden charm pentaquarks to the relevant channels}

The closeness of the $P_{c}$ (4312), $P_{c}(4440) / P_{c}$ (4457) to the $\Sigma_{c} \bar{D}$ and the $\Sigma_{c} \bar{D}^{*}$ thresholds, respectively, imply their hadronic molecular picture. Several studies [29,43,48,54, 67-69] have been implemented to explore their property in the molecular picture. The complete spectroscopy in the $\Sigma_{c}^{(*)} \bar{D}^{(*)}$ molecular picture should be three $\frac{1}{2}^{-}$pentaquarks, $\frac{3}{2}^{-}$pentaquarks and one $\frac{5}{2}^{-}$pentaquark. The heavy quark symmetry constraints that the number of parameters for the underlying dynamics is two, i.e. $C_{1}$ and $C_{3}$ defined as Eq.(4) of Ref. [67]. The corresponding channels and potentials in each channel are as follow. The three channels for $J^{P}=\frac{1}{2}^{-}$ are $\Sigma_{c} \bar{D}, \Sigma_{c} \bar{D}^{*}, \Sigma_{c}^{*} \bar{D}^{*}$ and the corresponding potential is
In the whole manuscript, we use the scattering amplitudes of pure contact results in Ref. [67] as inputs. The inclusion of the OPE and higher order contact potentials will not change the results significantly. When fit to the $J / \psi p$ invariant mass distribution of the $\Lambda_{b} \rightarrow J / \psi p K^{-}$process, two solutions can be found [48,67], i.e. Solution A and Solution B as denoted in Refs. [29,43]. In Solution A, the $P_{c}(4440)$ and the $P_{c}(4457)$ are assigned as $\frac{1}{2}^{-}$and $\frac{3}{2}^{-}$pentaquarks, respectively. In Solution B, they are interchanged. In the whole manuscript, we use the effective couplings with hard cutoff $1 \mathrm{GeV}$ in Ref. [67] as inputs. In the following, the effective couplings are collected in Tables 2 and 3.

\section{Appendix B: Cross section of the inclusive production of the $\Sigma_{c}^{(*)} \bar{D}^{(*)}$ pair in the LHC}

The property that the seven hidden charm pentaquarks, in the molecular picture, are very close the $\Sigma_{c}^{(*)} \bar{D}^{(*)}$ thresholds does not only lead to a large isospin breaking decay rate (such as the $J / \psi \Delta^{+}$channel [28]), but also could affect their production rates. That can be easily seen from Eq. (2), i.e. a small deviation of the thresholds will result in a large difference in the two-body propagator. If isospin symmetry works well, the inclusive production cross section of the $\Sigma_{c}^{(*)+} \bar{D}^{(*) 0}\left(\Sigma_{c}^{(*)+} D^{(*)-}\right)$ channel should equal to that of the $\Sigma_{c}^{(*)++} D^{(*)-}\left(\Sigma_{c}^{(*) 0} \bar{D}^{(*) 0}\right)$ channel. However, the initial $p p$ beam has a third isospin $I_{z}=+1$ which indicates that one would not expect the inclusive $I_{z}=+\frac{1}{2} \Sigma_{c}^{(*)+} \bar{D}^{(*) 0}$,

$V_{\frac{1}{2}}-=\left(\begin{array}{ccc}\frac{1}{3} C_{1}+\frac{2}{3} C_{3} & -\frac{2}{3 \sqrt{3}} C_{1}+\frac{2}{3 \sqrt{3}} C_{3} & \frac{1}{3} \sqrt{\frac{2}{3}} C_{1}-\frac{1}{3} \sqrt{\frac{2}{3}} C_{3} \\ -\frac{2}{3 \sqrt{3}} C_{1}+\frac{2}{3 \sqrt{3}} C_{3} & \frac{7}{9} C_{1}+\frac{2}{9} C_{3} & -\frac{1}{3} \sqrt{\frac{5}{3}} C_{1}+\frac{1}{3} \sqrt{\frac{5}{3}} C_{3} \\ \frac{1}{3} \sqrt{\frac{2}{3}} C_{1}-\frac{1}{3} \sqrt{\frac{2}{3}} C_{3}-\frac{1}{3} \sqrt{\frac{5}{3}} C_{1}+\frac{1}{3} \sqrt{\frac{5}{3}} C_{3} & \frac{5}{9} C_{1}+\frac{4}{9} C_{3}\end{array}\right)$.

The case for the $J^{P}=\frac{3}{2}^{-}$channel, the dynamical channels are $\Sigma_{c} \bar{D}^{*}, \Sigma_{c}^{*} \bar{D}, \Sigma_{c}^{*} \bar{D}^{*}$ and the corresponding potential is
$\Sigma_{c}^{(*)++} D^{(*)-}$ channels have the same inclusive cross section as those of the $I_{z}=-\frac{1}{2} \Sigma_{c}^{(*)+} D^{(*)-}, \Sigma_{c}^{(*) 0} \bar{D}^{(*) 0}$ channels.

$V_{\frac{3}{2}}{ }^{-}=\left(\begin{array}{ccc}\frac{1}{9} C_{1}+\frac{8}{9} C_{3} & -\frac{1}{3 \sqrt{3}} C_{1}+\frac{1}{3 \sqrt{3}} C_{3} & \frac{\sqrt{5}}{9} C_{1}-\frac{\sqrt{5}}{9} C_{3} \\ -\frac{1}{3 \sqrt{3}} C_{1}+\frac{1}{3 \sqrt{3}} C_{3} & \frac{1}{3} C_{1}+\frac{2}{3} C_{3} & -\frac{1}{3} \sqrt{\frac{5}{3}} C_{1}+\frac{1}{3} \sqrt{\frac{5}{3}} C_{3} \\ \frac{\sqrt{5}}{9} C_{1}-\frac{\sqrt{5}}{9} C_{3} & -\frac{1}{3} \sqrt{\frac{5}{3}} C_{1}+\frac{1}{3} \sqrt{\frac{5}{3}} C_{3} & \frac{5}{9} C_{1}+\frac{4}{9} C_{3}\end{array}\right)$.

There is only one channel, i.e. $\Sigma_{c}^{*} \bar{D}^{*}$ for the $J^{P}=\frac{5}{2}^{-}$channel and the potential is

$V_{5^{-}}-C_{3}$
Another reason is that we do not know the isospin information of the undetected particles for the inclusive processes. To illustrate this effect, we plot the cross sections of the inclusive $\Sigma_{c}^{(*)} \bar{D}^{(*)}$ channels explicitly in Figs. 6, 7, 8 and 9. From 
Table 2 The effective couplings of each channel for the three $\frac{1}{2}^{-}$, three $\frac{3}{2}^{-}$and one $\frac{5}{2}^{-}$pentaquarks in Solution A with hard cutoff $1 \mathrm{GeV}$. The second row is the corresponding pole positions. These values are taken from Ref. [67]

\begin{tabular}{lllll}
\hline$g_{\text {eff }}\left(\frac{1}{2}^{-}\right)$ & Pole $(\mathrm{MeV})$ & $\Sigma_{c} \bar{D}$ & $\Sigma_{c} \bar{D}^{*}$ & $\Sigma_{c}^{*} \bar{D}^{*}$ \\
\hline$P_{c}(4312)$ & $4314.7-i 3.4$ & $2.63+i 0.33$ & $0.77+i 0.1$ & $0.44+i 0.05$ \\
$P_{c}(4440)$ & $4439.8-i 7.2$ & $0.15+i 0.35$ & $3.74+i 0.46$ & $-0.75+i 0.3$ \\
$P_{c}\left(\frac{1}{2}^{-}\right)$ & $4497.4-i 6.9$ & $0.21+i 0.16$ & $-0.06-i 0.27$ & $4.09+i 0.24$ \\
\hline$g_{\mathrm{eff}}\left(\frac{3}{2}^{-}\right)$ & Pole $(\mathrm{MeV})$ & $\Sigma_{c} \bar{D}^{*}$ & $\Sigma_{c}^{*} \bar{D}$ & $\Sigma_{c}^{*} \bar{D}^{*}$ \\
\hline$P_{c}(4380)$ & $4377.2-i 6.1$ & $0.50+i 0.25$ & $-2.75-i 0.05$ & $-0.95+i 0.16$ \\
$P_{c}(4457)$ & $4459.3-i 3.0$ & $2.06+i 0.44$ & $0.05-i 0.09$ & $-0.78+i 0.32$ \\
$P_{c}\left(\frac{3}{2}^{-}\right)$ & $4506.9-i 18.6$ & $0.37+i 0.32$ & $-0.26-i 0.35$ & $-3.63-i 0.85$ \\
\hline$g_{\mathrm{eff}}\left(\frac{5}{2}^{-}\right)$ & Pole $(\mathrm{MeV})$ & $\Sigma_{c}^{*} \bar{D}^{*}$ & & \\
\hline$P_{c}\left(\frac{5}{2}^{-}\right)$ & $4526.7-i 13.6$ & & $2.18+i 1.29$ & \\
\hline
\end{tabular}

Table 3 The caption is analogous to that of Table 2 but for Solution B. These values are taken from Ref. [67]

\begin{tabular}{llllr}
\hline$g_{\text {eff }}\left(\frac{1}{2}^{-}\right)$ & Pole $(\mathrm{MeV})$ & $\Sigma_{c} \bar{D}$ & $\Sigma_{c} \bar{D}^{*}$ & $\Sigma_{c}^{*} \bar{D}^{*}$ \\
\hline$P_{c}(4312)$ & $4312.0-i 4.8$ & $2.92+i 0.43$ & $-0.77+i 0.07$ & $-0.51+i 0.08$ \\
$P_{c}(4457)$ & $4466.9-i 7.1$ & $0.08+i 0.41$ & $-2.02-i 1.58$ & $-0.32-i 0.28$ \\
$P_{c}\left(\frac{1}{2}^{-}\right)$ & $4530.5-i 9.7$ & $0.03+i 0.20$ & $0.04-i 0.18$ & $-1.59-i 1.67$ \\
\hline$g_{\mathrm{eff}}\left(\frac{3}{2}^{-}\right)$ & Pole $(\mathrm{MeV})$ & $\Sigma_{c} \bar{D}^{*}$ & $\Sigma_{c}^{*} \bar{D}$ & $\Sigma_{c}^{*} \bar{D}^{*}$ \\
\hline$P_{c}(4380)$ & $4374.0-i 5.9$ & $0.58-i 0.20$ & $3.05+i 0.10$ & $-0.87+i 0.18$ \\
$P_{c}(4440)$ & $4441.6-i 4.6$ & $3.63+i 0.32$ & $0.03+i 0.08$ & $-2.51-i 1.10$ \\
$P_{c}\left(\frac{3}{2}^{-}\right)$ & $4522.2-i 15.1$ & $0.00-i 0.31$ & $0.20+i 0.38$ & \\
\hline$g_{\mathrm{eff}}\left(\frac{5}{2}^{-}\right)$ & Pole $(\mathrm{MeV})$ & $\Sigma_{c}^{*} \bar{D}^{*}$ & & \\
\hline$P_{c}\left(\frac{5}{2}^{-}\right)$ & $4500.5-i 4.3$ & $3.95+i 0.07$ & & \\
\hline
\end{tabular}

the figures, one can also see the relation of the production rates for all the $\Sigma_{c}^{(*)} \bar{D}^{(*)}$ channels

$$
\sigma\left(\Sigma_{c}^{*} \bar{D}\right)>\sigma\left(\Sigma_{c} \bar{D}\right)>\sigma\left(\Sigma_{c} \bar{D}^{*}\right) \simeq \sigma\left(\Sigma_{c}^{*} \bar{D}^{*}\right)
$$



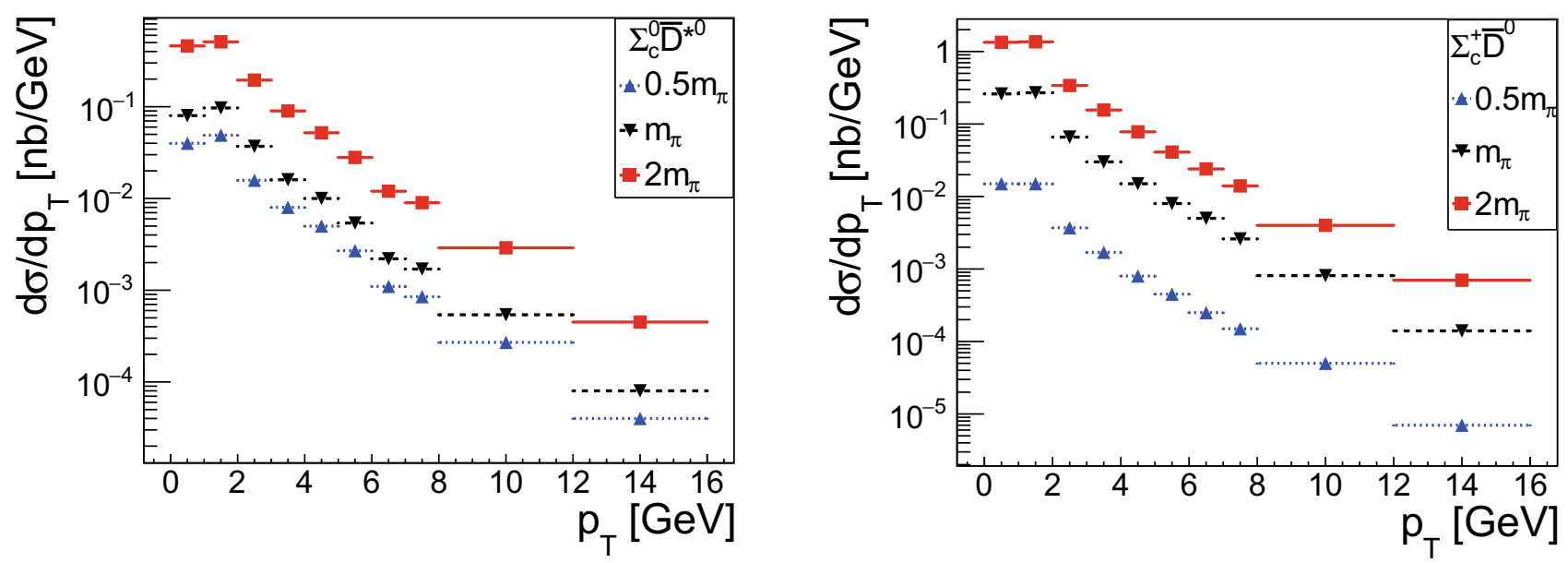

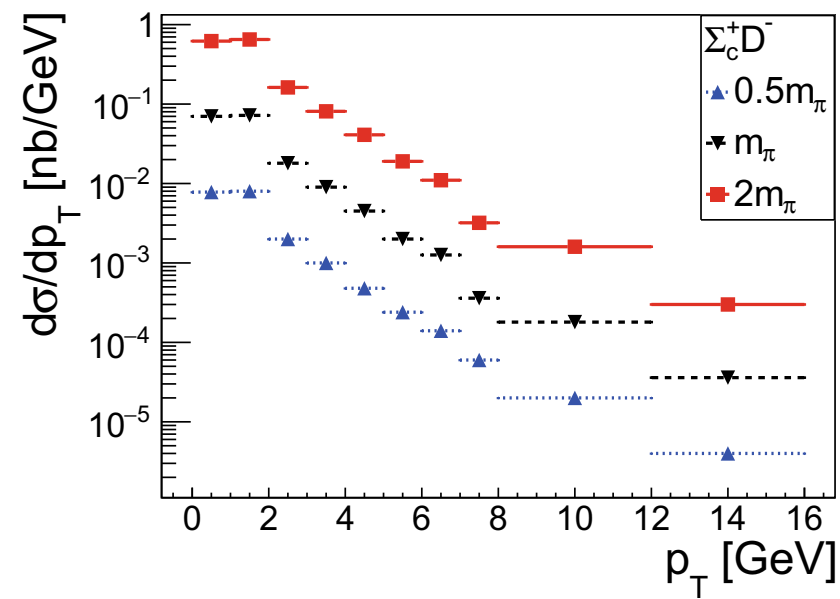

Fig. 6 The cross section of the inclusive production of the $\Sigma_{c} \bar{D}$ pairs in $p p$ collisions at center-of-mass energy $\sqrt{s}=7 \mathrm{TeV}$. The black inverted triangle, blue triangle, red box points are for the relative three momentum smaller than $0.5 m_{\pi}, m_{\pi}$ and $2 m_{\pi}$, respectively. As shown

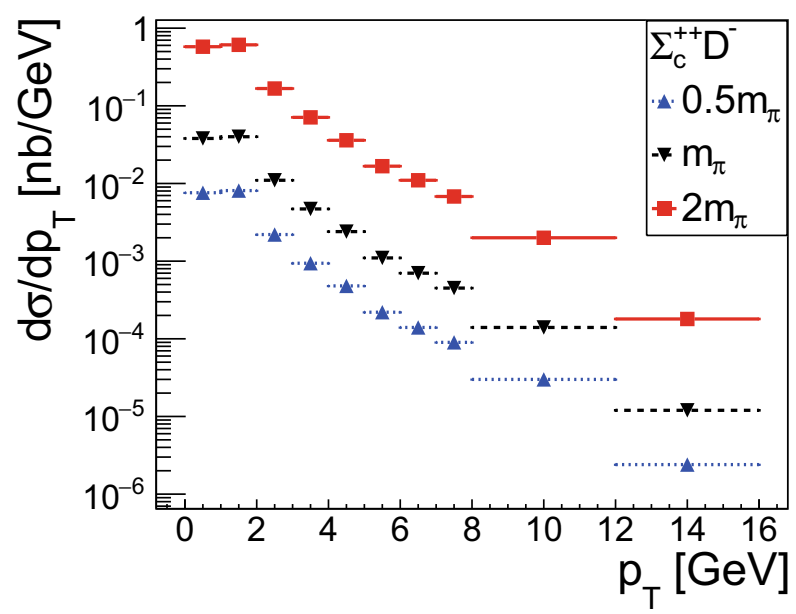

in the figures, there are significant isospin deviation for the inclusive productions of the $\Sigma_{c} \bar{D}$ pairs. In our calculation, we use $m_{\pi}$ to estimate the prompt cross section 

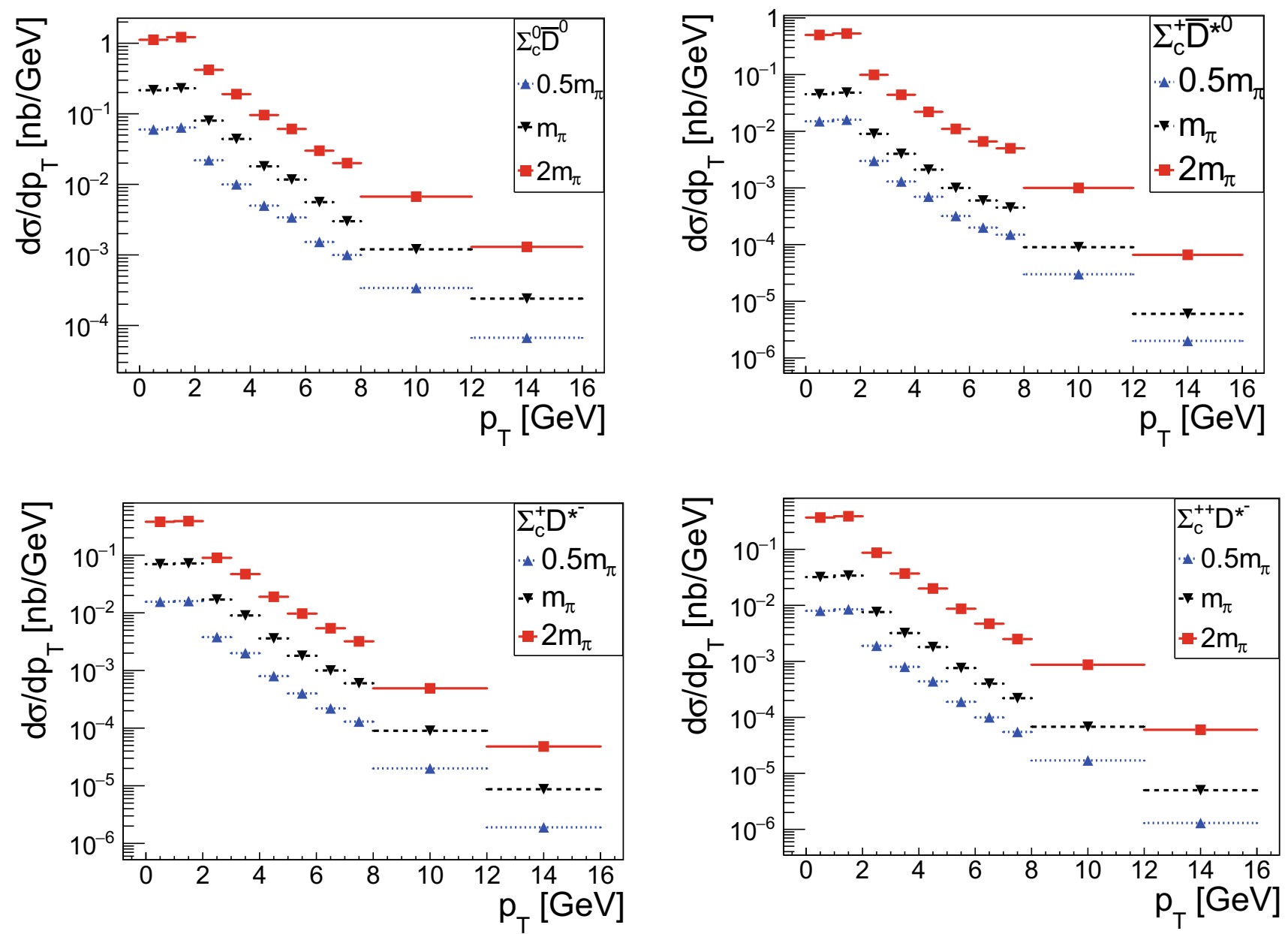

Fig. 7 The caption is analogous to that of Fig. 6, but for the $\Sigma_{c} \bar{D}^{*}$ channel 

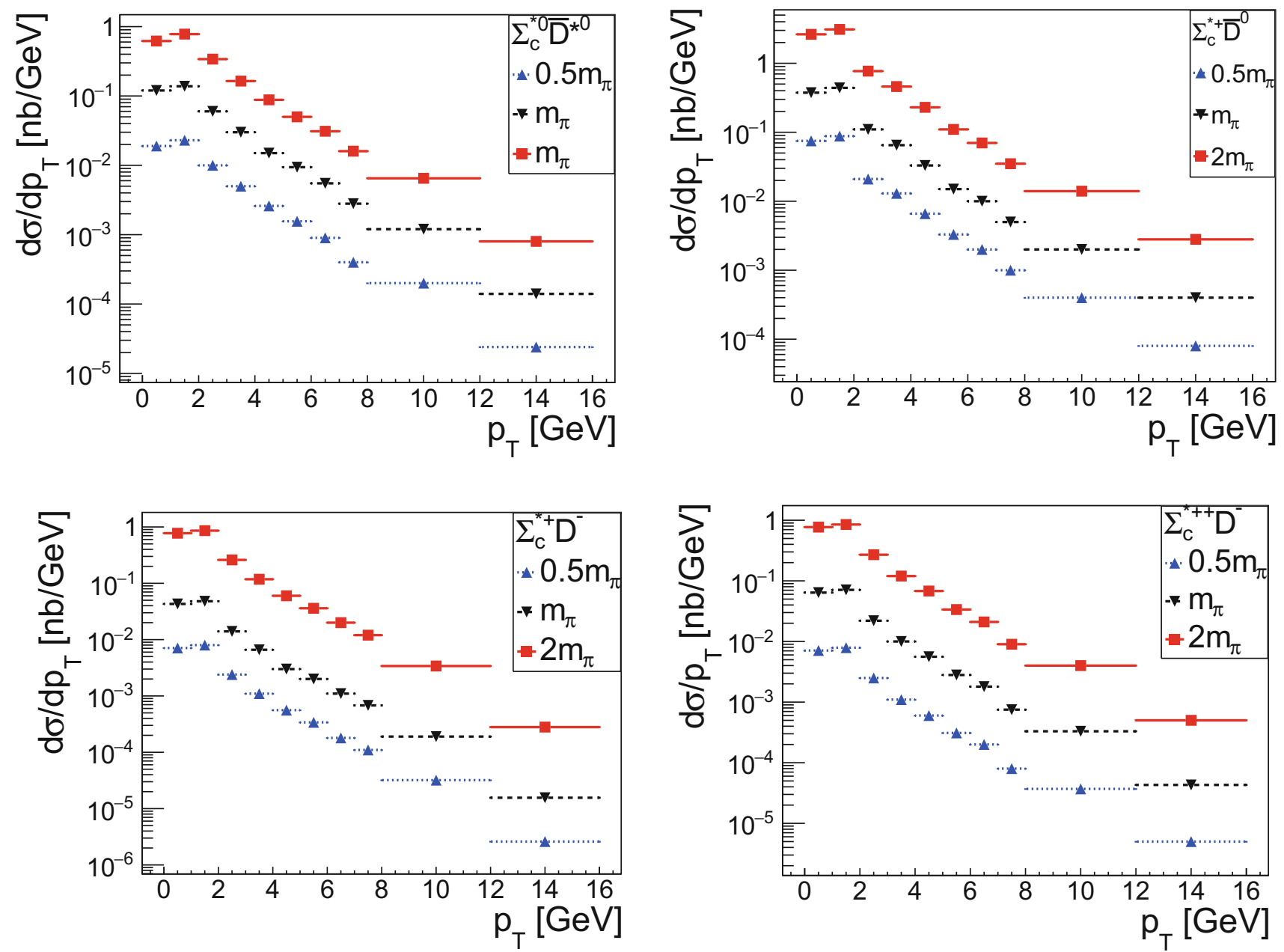

Fig. 8 The caption is analogous to that of Fig. 6, but for the $\Sigma_{c}^{*} \bar{D}$ channel 

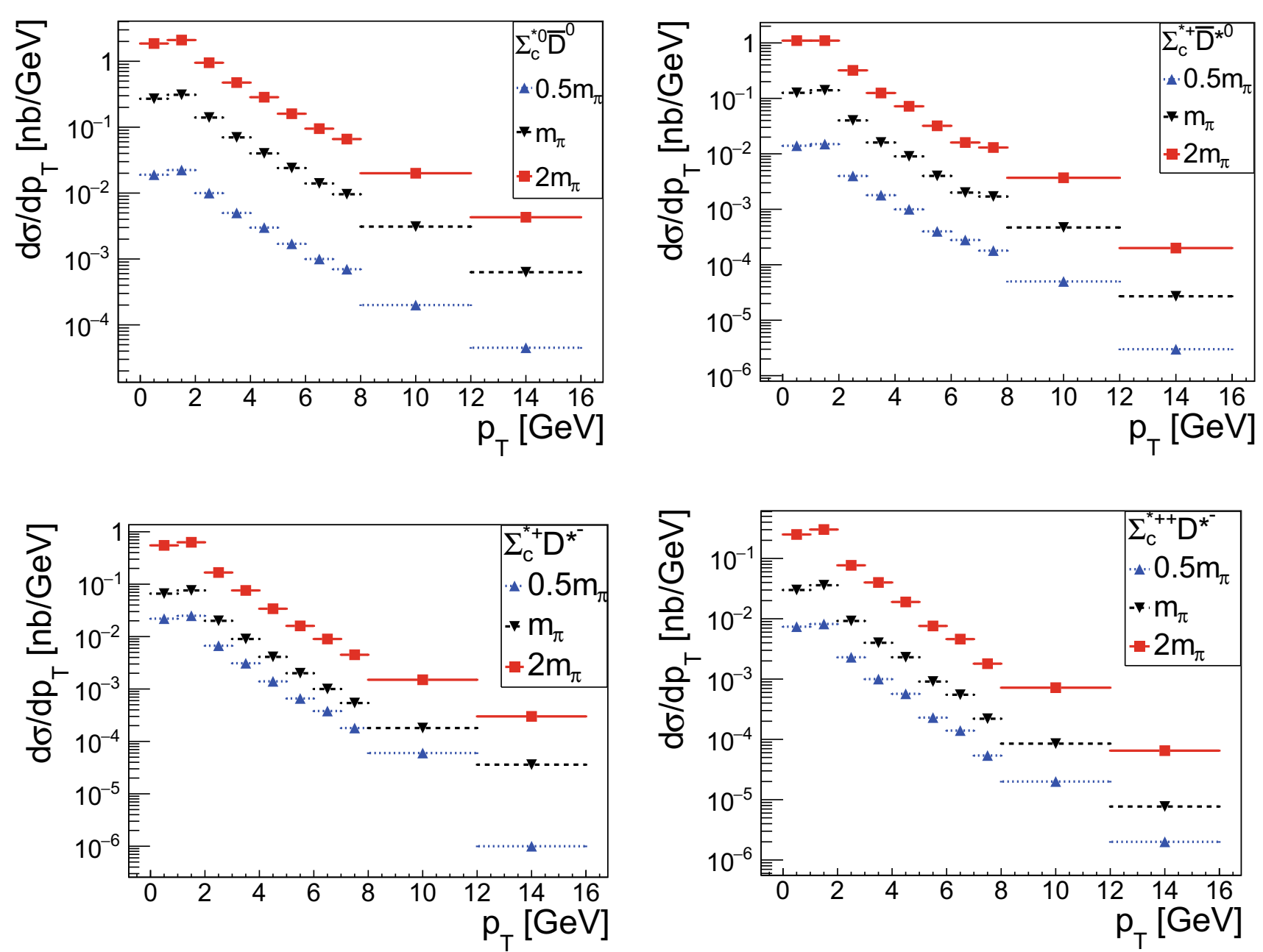

Fig. 9 The caption is analogous to that of Fig. 6, but for the $\Sigma_{c}^{*} \bar{D}^{*}$ channel 


\section{References}

1. R.L. Jaffe, Phys. Rev. D 15, 281 (1977)

2. B. Aubert et al. (BaBar), Phys. Rev. Lett. 90, 242001 (2003). arXiv:hep-ex/0304021

3. S.K. Choi et al. (Belle), Phys. Rev. Lett. 91, 262001 (2003). arXiv:hep-ex/0309032

4. S.L. Olsen, T. Skwarnicki, D. Zieminska, Rev. Mod. Phys. 90(1), 015003 (2018). arXiv:1708.04012 [hep-ph]

5. C.Z. Yuan, S.L. Olsen, Nat. Rev. Phys. 1(8), 480-494 (2019). arXiv:2001.01164 [hep-ex]

6. H.X. Chen, W. Chen, X. Liu, S.L. Zhu, Phys. Rep. 639, 1-121 (2016). arXiv:1601.02092 [hep-ph]

7. H.X. Chen, W. Chen, X. Liu, Y.R. Liu, S.L. Zhu, Rep. Prog. Phys. 80(7), 076201 (2017). arXiv:1609.08928 [hep-ph]

8. A. Esposito, A. Pilloni, A.D. Polosa, Phys. Rep. 668, 1-97 (2017). arXiv:1611.07920 [hep-ph]

9. A. Hosaka, T. Iijima, K. Miyabayashi, Y. Sakai, S. Yasui, PTEP 2016(6), 062 C01 (2016). arXiv:1603.09229 [hep-ph]

10. Y. Dong, A. Faessler, V.E. Lyubovitskij, Prog. Part. Nucl. Phys. 94, 282-310 (2017)

11. F.K. Guo, C. Hanhart, U.-G. Meißner, Q. Wang, Q. Zhao, B.S.Zou, Rev. Mod. Phys. 90(1), 015004 (2018). arXiv:1705.00141 [hep-ph]

12. A. Cerri, V.V. Gligorov, S. Malvezzi, J. Martin Camalich, J. Zupan, S. Akar, J. Alimena, B.C. Allanach, W. Altmannshofer, L. Anderlini et al., CERN Yellow Rep. Monogr. 7, 867-1158 (2019). arXiv:1812.07638 [hep-ph]

13. E. Kou et al. (Belle-II), PTEP 2019(12), 123 C01 (2019). arXiv:1808.10567 [hep-ex] [Erratum: PTEP 2020(2), 029201 (2020)]

14. N. Brambilla, S. Eidelman, C. Hanhart, A. Nefediev, C.P. Shen, C.E. Thomas, A. Vairo, C.Z. Yuan, Phys. Rep. 873, 1-154 (2020). arXiv:1907.07583 [hep-ex]

15. F.K. Guo, X.H. Liu, S. Sakai, Prog. Part. Nucl. Phys. 112, 103757 (2020). arXiv:1912.07030 [hep-ph]

16. Y.R. Liu, H.X. Chen, W. Chen, X. Liu, S.L. Zhu, Prog. Part. Nucl. Phys. 107, 237-320 (2019). arXiv:1903.11976 [hep-ph]

17. R. Aaij et al. (LHCb), Phys. Rev. Lett. 115, 072001 (2015). arXiv:1507.03414 [hep-ex]

18. J.J. Wu, R. Molina, E. Oset, B.S. Zou, Phys. Rev. Lett. 105, 232001 (2010). arXiv:1007.0573 [nucl-th]

19. W.L. Wang, F. Huang, Z.Y. Zhang, B.S. Zou, Phys. Rev. C 84, 015203 (2011). arXiv: 1101.0453 [nucl-th]

20. J.J. Wu, T.S.H. Lee, B.S. Zou, Phys. Rev. C 85, 044002 (2012). arXiv:1202.1036 [nucl-th]

21. C.W. Xiao, J. Nieves, E. Oset, Phys. Rev. D 88, 056012 (2013). arXiv:1304.5368 [hep-ph]

22. M. Karliner, J.L. Rosner, Phys. Rev. Lett. 115(12), 122001 (2015). arXiv:1506.06386 [hep-ph]

23. Z.C. Yang, Z.F. Sun, J. He, X. Liu, S.L. Zhu, Chin. Phys. C 36, 6-13 (2012). arXiv:1105.2901 [hep-ph]

24. S.G. Yuan, K.W. Wei, J. He, H.S. Xu, B.S. Zou, Eur. Phys. J. A 48, 61 (2012). arXiv:1201.0807 [nucl-th]

25. R. Aaij et al. (LHCb), Phys. Rev. Lett. 122(22), 222001 (2019). arXiv:1904.03947 [hep-ex]

26. H.X. Chen, W. Chen, S.L. Zhu, Phys. Rev. D 100(5), 051501 (2019). arXiv:1903.11001 [hep-ph]

27. R. Chen, Z.F. Sun, X. Liu, S.L. Zhu, Phys. Rev. D 100(1), 011502 (2019). arXiv:1903.11013 [hep-ph]

28. F.K. Guo, H.J. Jing, U.-G. Meißner, S. Sakai, Phys. Rev. D 99(9), 091501 (2019). arXiv:1903.11503 [hep-ph]

29. M.Z. Liu, Y.W. Pan, F.Z. Peng, M. Sánchez Sánchez, L.S. Geng, A. Hosaka, M. Pavon Valderrama, Phys. Rev. Lett. 122(24), 242001 (2019). arXiv:1903.11560 [hep-ph]

30. J. He, Eur. Phys. J. C 79(5), 393 (2019). arXiv:1903.11872 [hep-ph]
31. Z.H. Guo, J.A. Oller, Phys. Lett. B 793, 144-149 (2019). arXiv:1904.00851 [hep-ph]

32. Y. Shimizu, Y. Yamaguchi, M. Harada, arXiv:1904.00587 [hep-ph]

33. C.J. Xiao, Y. Huang, Y.B. Dong, L.S. Geng, D.Y. Chen, Phys. Rev. D 100(1), 014022 (2019). arXiv:1904.00872 [hep-ph]

34. C.W. Xiao, J. Nieves, E. Oset, Phys. Rev. D 100(1), 014021 (2019). arXiv:1904.01296 [hep-ph]

35. F.L. Wang, R. Chen, Z.W. Liu, X. Liu, Phys. Rev. C 101(2), 025201 (2020). arXiv:1905.03636 [hep-ph]

36. L. Meng, B. Wang, G.J. Wang, S.L. Zhu, Phys. Rev. D 100(1), 014031 (2019). arXiv:1905.04113 [hep-ph]

37. J.J. Wu, T.S.H. Lee, B.S. Zou, Phys. Rev. C 100(3), 035206 (2019). arXiv: 1906.05375 [nucl-th]

38. C.W. Xiao, J. Nieves, E. Oset, Phys. Lett. B 799, 135051 (2019). arXiv:1906.09010 [hep-ph]

39. M.B. Voloshin, Phys. Rev. D 100(3), 034020 (2019). arXiv:1907.01476 [hep-ph]

40. S. Sakai, H.J. Jing, F.K. Guo, Phys. Rev. D 100(7), 074007 (2019). arXiv:1907.03414 [hep-ph]

41. Z.G. Wang, X. Wang, Chin. Phys. C 44, 103102 (2020). arXiv:1907.04582 [hep-ph]

42. Y. Yamaguchi, H. García-Tecocoatzi, A. Giachino, A. Hosaka, E. Santopinto, S. Takeuchi, M. Takizawa, Phys. Rev. D 101(9), 091502 (2020). arXiv:1907.04684 [hep-ph]

43. M.Z. Liu, T.W. Wu, M. Sánchez Sánchez, M.P. Valderrama, L.S. Geng, J.J. Xie, Phys. Rev. D 103(5), 054004 (2021). arXiv:1907.06093 [hep-ph]

44. Y.H. Lin, B.S. Zou, Phys. Rev. D 100(5), 056005 (2019). arXiv:1908.05309 [hep-ph]

45. B. Wang, L. Meng, S.L. Zhu, JHEP 11, 108 (2019). arXiv:1909.13054 [hep-ph]

46. T. Gutsche, V.E. Lyubovitskij, Phys. Rev. D 100(9), 094031 (2019). arXiv:1910.03984 [hep-ph]

47. T.J. Burns, E.S. Swanson, Phys. Rev. D 100(11), 114033 (2019). arXiv:1908.03528 [hep-ph]

48. M.L. Du, V. Baru, F.K. Guo, C. Hanhart, U.-G. Meißner, J.A. Oller, Q. Wang, Phys. Rev. Lett. 124(7), 072001 (2020). arXiv:1910.11846 [hep-ph]

49. G.J. Wang, L.Y. Xiao, R. Chen, X.H. Liu, X. Liu, S.L. Zhu, Phys. Rev. D 102(3), 036012 (2020). arXiv:1911.09613 [hep-ph]

50. H. Xu, Q. Li, C.H. Chang, G.L. Wang, Phys. Rev. D 101(5), 054037 (2020). arXiv:2001.02980 [hep-ph]

51. S.Q. Kuang, L.Y. Dai, X.W. Kang, D.L. Yao, Eur. Phys. J. C 80(5), 433 (2020). arXiv:2002.11959 [hep-ph]

52. F.Z. Peng, M.Z. Liu, M. Sánchez Sánchez, M. PavonValderrama, Phys. Rev. D 102, 114020 (2020). arXiv:2004.05658 [hep-ph]

53. F.Z. Peng, J.X. Lu, M. Sánchez Sánchez, M.J. Yan, M. Pavon Valderrama, Phys. Rev. D 103(1), 014023 (2021). arXiv:2007.01198 [hep-ph]

54. C.W. Xiao, J.X. Lu, J.J. Wu, L.S. Geng, Phys. Rev. D 102(5), 056018 (2020). arXiv:2007.12106 [hep-ph]

55. X.K. Dong, F.K. Guo, B.S. Zou, Prog. Phys. 41, 65-93 (2021).

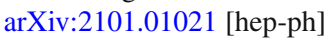

56. F.Z. Peng, M. Sánchez Sánchez, M.J. Yan, M. Pavon Valderrama, arXiv:2101.07213 [hep-ph]

57. A. Ali, A.Y. Parkhomenko, Phys. Lett. B 793, 365-371 (2019). arXiv:1904.00446 [hep-ph]

58. R. Zhu, X. Liu, H. Huang, C.F. Qiao, Phys. Lett. B 797, 134869 (2019). arXiv:1904.10285 [hep-ph]

59. Z.G. Wang, Int. J. Mod. Phys. A 35(01), 2050003 (2020). arXiv:1905.02892 [hep-ph]

60. J.F. Giron, R.F. Lebed, C.T. Peterson, JHEP 05, 061 (2019). arXiv:1903.04551 [hep-ph]

61. J.B. Cheng, Y.R. Liu, Phys. Rev. D 100(5), 054002 (2019). arXiv:1905.08605 [hep-ph] 
62. F. Stancu, Eur. Phys. J. C 79(11), 957 (2019). arXiv:1902.07101 [hep-ph]

63. U. Özdem, Eur. Phys. J. C 81(4), 277 (2021). arXiv:2102.01996 [hep-ph]

64. M.I. Eides, V.Y. Petrov, M.V. Polyakov, Phys. Rev. D 93(5), 054039 (2016). arXiv:1512.00426 [hep-ph]

65. M.I. Eides, V.Y. Petrov, M.V. Polyakov, Mod. Phys. Lett. A 35(18), 2050151 (2020). arXiv:1904.11616 [hep-ph]

66. J. Ferretti, E. Santopinto, M. Naeem Anwar, M.A. Bedolla, Phys. Lett. B 789, 562-567 (2019). arXiv:1807.01207 [hep-ph]

67. M. L. Du, V. Baru, F. K. Guo, C. Hanhart, U.-G. Meißner, J. A. Oller, Q. Wang, JHEP 08, 157 (2021). arXiv:2102.07159 [hep-ph]

68. Y.W. Pan, M.Z. Liu, F.Z. Peng, M. Sánchez Sánchez, L.S. Geng, M. Pavon Valderrama, Phys. Rev. D 102(1), 011504 (2020). arXiv:1907.11220 [hep-ph]

69. M. Pavon Valderrama, Phys. Rev. D 100(9), 094028 (2019). arXiv:1907.05294 [hep-ph]

70. S.X. Nakamura, Phys. Rev. D 103, 111503 (2021). arXiv:2103.06817 [hep-ph]

71. J. Alwall, M. Herquet, F. Maltoni, O. Mattelaer, T. Stelzer, JHEP 06, 128 (2011). arXiv:1106.0522 [hep-ph]

72. T. Sjostrand, S. Mrenna, P.Z. Skands, Comput. Phys. Commun. 178, 852-867 (2008). arXiv:0710.3820 [hep-ph]

73. P. Artoisenet, E. Braaten, Phys. Rev. D 81, 114018 (2010). arXiv:0911.2016 [hep-ph]

74. P. Artoisenet, E. Braaten, Phys. Rev. D 83, 014019 (2011). arXiv:1007.2868 [hep-ph]
75. F.K. Guo, U.-G. Meißner, W. Wang, Z. Yang, JHEP 05, 138 (2014). arXiv:1403.4032 [hep-ph]

76. F.K. Guo, U.-G. Meißner, W. Wang, Commun. Theor. Phys. 61, 354-358 (2014). arXiv:1308.0193 [hep-ph]

77. F.K. Guo, U.-G. Meißner, W. Wang, Z. Yang, Eur. Phys. J. C 74(9), 3063 (2014). arXiv:1402.6236 [hep-ph]

78. C. Bignamini, B. Grinstein, F. Piccinini, A.D. Polosa, C. Sabelli, Phys. Rev. Lett. 103, 162001 (2009). arXiv:0906.0882 [hep-ph]

79. Y. Jin, S.Y. Li, Y.R. Liu, L. Meng, Z.G. Si, X.F. Zhang, Chin. Phys. C 41(8), 083106 (2017). arXiv:1610.04411 [hep-ph]

80. M. Albaladejo, F.K. Guo, C. Hanhart, U.-G. Meißner, J. Nieves, A. Nogga, Z. Yang, Chin. Phys. C 41(12), 121001 (2017). arXiv:1709.09101 [hep-ph]

81. W. Wang, Chin. Phys. C 42(4), 043103 (2018). arXiv:1709.10382 [hep-ph]

82. E. Braaten, L.P. He, K. Ingles, Phys. Rev. D 100(9), 094024 (2019). arXiv:1811.08876 [hep-ph]

83. A.L. Guerrieri, F. Piccinini, A. Pilloni, A.D. Polosa, Phys. Rev. D 90(3), 034003 (2014). arXiv:1405.7929 [hep-ph]

84. R. Tieulent (ALICE), Acta Phys. Pol. B 42, 1655-1662 (2011). arXiv:1107.4509 [hep-ex]

85. S. Acharya et al. (ALICE), JHEP 04, 108 (2018). arXiv: 1712.09581 [nucl-ex]

86. M. Cacciari, S. Frixione, N. Houdeau, M.L. Mangano, P. Nason, G. Ridolfi, JHEP 10, 137 (2012). arXiv:1205.6344 [hep-ph]

87. G. Aad et al. (ATLAS), Eur. Phys. J. C 71, 1630 (2011). arXiv:1101.2185 [hep-ex] 